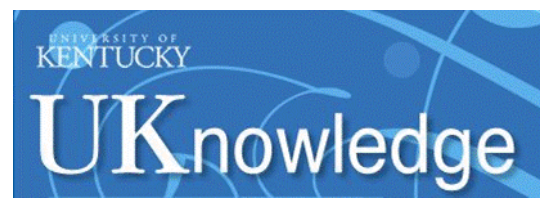

University of Kentucky

UKnowledge

4-2013

\title{
The Mathematics of Drug Dose Individualization Should Be Built With Random-Effects Linear Models
}

\author{
Francisco J. Diaz \\ University of Kansas Medical Center \\ Jose de Leon \\ University of Kentucky, jdeleon@uky.edu
}

Follow this and additional works at: https://uknowledge.uky.edu/psychiatry_facpub

Part of the Psychiatry and Psychology Commons

Right click to open a feedback form in a new tab to let us know how this document benefits you.

\section{Repository Citation}

Diaz, Francisco J. and de Leon, Jose, "The Mathematics of Drug Dose Individualization Should Be Built With Random-Effects Linear Models" (2013). Psychiatry Faculty Publications. 16.

https://uknowledge.uky.edu/psychiatry_facpub/16

This Letter to the Editor is brought to you for free and open access by the Psychiatry at UKnowledge. It has been accepted for inclusion in Psychiatry Faculty Publications by an authorized administrator of UKnowledge. For more information, please contact UKnowledge@lsv.uky.edu. 


\section{The Mathematics of Drug Dose Individualization Should Be Built With Random- Effects Linear Models}

\section{Digital Object Identifier (DOI)}

http://dx.doi.org/10.1097/FTD.0b013e318283e3c6

\section{Notes/Citation Information}

Published in Therapeutic Drug Monitoring, v. 35, issue 2, p. 276-277.

This is a non-final version of an article published in final form in "Diaz FJ, de Leon J. The mathematics of drug dose individualization should be built with random effects linear models (letter to editor).

Therapeutic Drug Monitoring 35(2):276-7, 2013. doi: 10.1097/FTD.0b013e318283e3c6." 


\section{Letter to the Editor}

The mathematics of drug dose individualization should be built with random effects linear models

Francisco J. Diaz, Ph.D.\#, Jose de Leon, M.D.*

"Department of Biostatistics, The University of Kansas Medical Center, Kansas City, KS, United States.

*University of Kentucky Mental Health Research Center at Eastern State Hospital, Lexington, KY, United States, and Psychiatry and Neurosciences Research Group (CTS-549), Institute of Neurosciences, University of Granada, Granada, Spain.

Corresponding author: Francisco J. Diaz, Ph.D., Department of Biostatistics, Mail Stop 1026, 3901 Rainbow Blvd., Kansas City, KS 66160, United States. Phone: (913) 945-7006. Fax: (913) 588-0252. Email:fdiaz@kumc.edu

Sources of funding: Dr. Diaz's writing of this letter was supported by Frontiers: The Heartland Institute for Clinical and Translational Research CTSA UL1TR000001 (awarded to the University of Kansas Medical Center). The contents are solely the responsibility of the authors and do not necessarily represent the official views of the NIH.

Acknowledgements: The authors acknowledge Mrs. Rosemary Morrow, at the Department of Biostatistics of the University of Kansas Medical Center, who helped in editing this letter.

Conflicts of interest: None declared.

Keywords: Dose individualization; empirical Bayesian methods; omega optimum dose; random effects linear models; target concentration intervention. 
Sir,

We are delighted at the publication of Holford and Buclin's article ${ }^{1}$, which proposes a general theoretical framework for the clinical problem of individualizing drug doses. The essence of their proposal is that a careful assessment of between-patient and within-patient variability in the pharmacokinetic or pharmacodynamic response of interest should be made to decide whether a target concentration intervention ( $\mathrm{TCl}$ ) is appropriate, and should be made prior to formulating a $\mathrm{TCl}$. Although Holford and Buclin do not give details about how these variabilities should be computationally combined with a particular patient's drug plasma concentrations and covariate values in order to implement a $\mathrm{TCl}$ in practice, their description of criteria to determine whether or not a $\mathrm{TCl}$ is necessary is an important contribution. Another reason for our enthusiasm is that Holford and Buclin's framework is conceptually equivalent to a rigorous mathematical formulation of dose individualization that we developed in several prior publications. . $^{2,3}$ In fact, we can say that if a pharmacologist is willing to computationally implement Holford and Buclin's ideas in a TCl by using standard statistical software, or to rigorously derive further practical and methodological consequences of these ideas for therapeutic drug monitoring (TDM), then he/she may want to read our publications as a next step. ${ }^{2,3,4}$

Each and every pharmacokinetic concept described in Holford and Buclin's article has a corresponding and equivalently defined concept in our mathematical formulation. Table 1 describes the equivalence between Holford and Buclin's concepts and some of our concepts. We hope that this table serves as a guideline for those who want to proceed to read our more mathematically demanding papers. ${ }^{2,3} \mathrm{~A}$ friendly introduction for clinicians and pharmacologists, and epistemological consequences of the approach are in reference 4. Our mathematical formulation of dose individualization is based on random effects linear models, a family of statistical models that is well known and widely used by professional statisticians, but that, unfortunately, is not well known by pharmacologists. We celebrate that Holford and Buclin seem to have been inspired by random-effects ideas. This is suggested by the fact that they cite a work from the Sheiner School in their paper, ${ }^{5}$ a school that for decades have advocated the use of random-effects models (also called mixed regression models) for developing dose individualization methods. The Sheiner School, however, places emphasis on random-effects nonlinear models, not linear models, which is a reason to consider Holford and Buclin's ideas a significant conceptual jump.

The fact that other authors have independently developed ideas about dose individualization that are essentially equivalent to ours confirms that random effects linear models are the natural mathematical language of a pharmacological theory and practice of dose individualization. ${ }^{4}$ Our mathematical formulation based on these models provides answers to important questions that are not addressed in Holford and Buclin's article. For instance, 1) How can we compute an optimal dose for a particular patient, that is, what is the optimal way of combining steady-state concentrations from an individual patient with his/her demographic, clinical or genetic covariates in order to obtain an appropriate dose for the patient?; 2) What is the minimum number of blood samples at steady state that are needed from a patient in order to compute an optimal dose for that patient?; and 3) Do dose individualization methods currently used in TDM produce optimal doses?. After laying out the term "optimal dose" with 
a concept that we have called omega-optimality, we have demonstrated through both decisiontheoretical arguments and computer simulations that a traditional TDM method of dose individualization that is advocated in many pharmacology textbooks does not produce optimal doses (see also footnote $\mathrm{h}$ to Table 1). ${ }^{3}$ Also, we have computed a table that provides the minimum number of blood samples that are needed from individual patients in order to obtain an omega-optimum dose for a high percentage of the patients in the population. ${ }^{2}$ Finally, the basic tenet of the Sheiner School, that an empirical Bayesian approach should be used to amalgamate all the available information from a patient with prior population information in order to search for an optimum dose for the patient, can be shown to be particularly valid (and easy to implement computationally) when random effects linear models are used. ${ }^{2,3}$

\section{References}

1. Holford, NHG , Buclin, T. Safe and effective variability-a criterion for dose individualization. Ther Drug Monit. 2012; 34:565-568.

2. Diaz FJ, Rivera TE, Josiassen RC, et al. Individualizing drug dosage by using a random intercept linear model. Stat Med. 2007;26:2052-2073.

3. Diaz, FJ, Cogollo, M, Spina, E, et al. Drug dosage individualization based on a random-effects linear model. J Biopharm Stat. 2012;22:463-484.

4. Diaz FJ, Yeh, HG, de Leon J. Role of statistical random-effects linear models in personalized medicine. Curr Pharmacogenomics Person Med. 2012;10:22-32.

5. Sheiner LB, Rosenburg B, Marathe VV. Estimation of population characteristics of pharmacokinetic parameters from routine clinical data. J Pharmacokinet Biopharm. 1977;5:445479. 
Table 1. List of concepts that Holford and Buclin ${ }^{1}$ use to build their approach to dose individualization, and the conceptually equivalent concepts that Diaz et al. 2,3 use to build their statistical model and clinical algorithm for target concentration intervention.

\begin{tabular}{|c|c|c|c|}
\hline Holford and Buclin's ${ }^{1}$ concept & $\underline{\text { Symbol used }}$ & Diaz et al.'s $\mathrm{s}^{2,3}$ equivalent concept ${ }^{\mathrm{b}}$ & ${\underline{S y m b o l}(\mathrm{~s}) \text { used }^{\mathrm{c}}}$ \\
\hline Target concentration & $\mathrm{TC}$ & Target concentration ${ }^{d}$ & $C_{0}$ \\
\hline Safe and effective concentration range & SECR & Target concentration range (TCR) & $\left(l_{1}, l_{2}\right)$ \\
\hline Unpredictable between-subject variability & $\mathrm{BSV}_{\mathrm{U}}$ & Between-subjects variance ${ }^{e}$ & $\sigma_{\alpha}^{2}$ \\
\hline Unpredictable within-subject variability & WSV $_{u}$ & Intra-individual random error & $\sigma_{\epsilon}^{2}$ \\
\hline Unpredictable variability & $\mathrm{PPV}_{U}$ & Total variance of log of steady-state plasma concentrations & $\sigma_{\alpha}^{2}+\sigma_{\epsilon}^{2}$ \\
\hline Predictable variability & $\mathrm{PPV}_{\mathrm{P}}$ & Combination of fixed effects and covariates ${ }^{f}$ & $\mu_{\alpha}+\boldsymbol{\beta}^{T} \boldsymbol{X}$ \\
\hline Safe and effective variability & SEV & $\begin{array}{l}\text { Fraction of maximum attainable probability } \\
\text { that patient reaches } \mathrm{TCR}^{\mathrm{g}}\end{array}$ & $\omega \times m, \omega \approx 1$ \\
\hline Individual target dose & ITD & Dose computed at second step of individualization algorithm ${ }^{\mathrm{h}}$ & $D_{2}$ \\
\hline Clearance & $\mathrm{CL}$ & Patient's concentration-to-dose ratio at steady state ${ }^{i}$ & $e^{\alpha+\beta^{T} X}$ \\
\hline
\end{tabular}

${ }^{a}$ Symbol used in Holford and Buclin's paper. ${ }^{1}$

${ }^{b}$ Some of the concepts used by Diaz et al. ${ }^{2,3}$ are standard concepts from the statistical literature on random effects linear models.

'Symbol(s) or formula used in Diaz et al.'s paper. ${ }^{2}$ 
${ }^{\mathrm{d}}$ Diaz et al. ${ }^{2,3}$ also computed an optimal target concentration, symbolized by $C_{0}^{*}$, when it is desired to reach a particular target concentration range. However, any desired target concentration can be input into their dose individualization algorithm, including the TC suggested in formula (1) of Holford and Buclin. ${ }^{1}$

'This is the variance of the random intercept, ${ }^{2}$ or in general, the compounded variances of the random effects in a more general formulation given in reference 3.

fIn the general mathematical formulation given in reference 3, covariates that have random effects on plasma concentrations also contribute to the predictable variability.

${ }^{g}$ Similarly to the SEV concept of Holford and Buclin, ${ }^{1}$ the maximum attainable probability of a patient reaching the TCR (symbolized with $m$ by Diaz et al. $\left.{ }^{2,3}\right)$ is essentially a function of both the SECR and WSV $\mathrm{W}_{u}$ or, equivalently, of $\left(l_{1}, l_{2}\right)$ and $\sigma_{\epsilon}^{2}$. Under a TCl, the exact relationship between $\omega \times m$ and $\mathrm{SEV}$ is $\omega \times m=1-2 \Phi\left(-\left(\log \sqrt{l_{2} / l_{1}}\right) / \mathrm{SEV}\right)$, where $\Phi$ is the standard normal cumulative distribution function.

${ }^{\text {h}} \mathrm{A}$ limitation of Holford and Buclin ${ }^{1}$ paper is they do not specifically describe how to compute the ITD from a particular patient's drug plasma concentration. However, they describe that the ITD is usually computed with a concentration produced in the patient by a group therapy dose (GTD). If an empirical Bayesian approach such as that proposed by Diaz et al. ${ }^{2,3}$ were used to carry out this computation, the ITD would correspond to $D_{2}$, the dose computed at the second step of Diaz et al.'s individualization algorithm. Depending on the particular values of BSV $u$ and WSV $\mathrm{W}_{u}$, and of SECR, this dose may or may not be optimal according to the omega-optimality criterion of Diaz et al. ${ }^{2,3}$ If it is not optimal, additional algorithm steps are needed to obtain optimality. In general, depending on both how the ITD is computed and the patient population characteristics, the ITD may or may not be optimal in the sense that the probability that a particular patient reaches the SER with such dose may or may not be as high as possible.

iDiaz et al. ${ }^{3,4}$ describe empirical and theoretical evidence from them and other authors demonstrating that their statistical model is essentially a model of clearance when the log of steady-state drug plasma concentrations is used as the dependent variable and the drug follows linear pharmacokinetics. 